\title{
Chipanas y orejones en la frontera oriental del Tawantinsuyu. Una propuesta desde la arqueología y la etnohistoria para los valles del norte de Salta, Argentina
}

Beatriz N. Ventura*

Fecha de recepción: 27 de junio de 2018. Fechas de aceptación: 28 de octubre de 2018

\section{Resumen}

Los valles orientales del norte de Salta formaron parte de la frontera oriental del Tawantinsuyu. En el sector de los valles de Iruya, Nazareno y Bacoya la ocupación tuvo objetivos principalmente económicos, organizándose un enclave con presencia de grupos jerarquizados. Las principales actividades en este sector del oriente de las Serranías de Santa Victoria fueron las minero-metalúrgicas; entre ellas, la producción de bienes suntuarios. En base al registro arqueológico de estos valles, y a documentación y cartografía histórica, consideramos que las piezas de metal características de estos valles fueron las chipanas. Estas pulseras y/o brazaletes fueron símbolos de prestigio de alta valoración dentro de la sociedad andina -en especial de la incaica. Estas piezas, de gran valor simbólico, sirvieron como elementos de intercambio, como regalos al Inca y a curacas y orejones en festividades, y fueron importantes objetos de transacciones, tanto en tiempos incaicos como durante la colonia temprana.

Chipanas and orejones in the Tawantinsuyu eastern frontier. An archaeological and ethnohistorical proposal for the valleys of northern Salta, Argentina

\footnotetext{
Abstract

The eastern valleys of northern Salta were part of the eastern border of Tawantinsuyu. In the sector of the Iruya, Nazareno and Bacoya valleys the occupation had economic objectives, comprising an enclave occupied by hierarchical groups. The mineral-metallurgical activities were among the main goals pursued in this eastern sector of the Serranías de Santa Victoria,

\section{Palabras clave}

ornamentos de prestigio metalurgia Tawantinsuyu Salta

Key words

prestigious goods metallurgy Tawantinsuyu Salta 
particularly the production of luxury goods. According to the archaeological record, and taking into consideration the available historic documentation and cartography, we surmise that the metal pieces that characterized the production in these valleys were the chipanas. These bracelets were considered symbols of prestige highly valued within Andean society -especially among the Incas. These pieces of great symbolic value were used as economic exchange goods, as presents for the Inca during festivities, as well as for curacas and orejones. They were also important objects for economic transactions during Inca and early colonial times.

\section{Introducción}

Los incas implementaron distintas estrategias en su intento de dominar a las poblaciones y de ocupar el amplio territorio que se extiende al oriente de los Andes. La franja sur oriental del Tawantinsuyu es uno de los sectores del territorio incaico que ha sido poco estudiado arqueológicamente, y el conocimiento que tenemos de él se sustenta en el valioso aporte de la documentación histórica. Principalmente sobre esa base, las ocupaciones de estos territorios se han atribuido a diferentes causas y a la explotación de ciertos recursos. Según esas fuentes, los conflictos militares requirieron el traslado de poblaciones en Samaipata y Oroncota, en el este boliviano (Alconini, 2004; Meyers y Combés, 2015). También el desarrollo agrícola de los fértiles valles de Cochabamba se logró gracias a grandes movimientos de poblaciones desde diversos sectores del Imperio (Wachtel, 1982), al igual que la explotación minero-metalúrgica en Saipuru (Pärssinen, 1992; Cruz y Guillot, 2010).

A lo largo de la frontera sur oriental el registro arqueológico que ha quedado de esas ocupaciones y la documentación histórica que las consigna son muy desiguales y su conocimiento es, en general, escaso. Las diversas formas del dominio estatal en esos espacios recién comienzan a percibirse en los últimos años al profundizar en el estudio documental y arqueológico de ciertos sectores, como lo muestran algunos de los sitios mencionados, pero aún estamos muy lejos de tener un panorama general de esa frontera. Es por eso que consideramos valioso nuestro aporte regional al poder complementar la información a través de ambos registros.

Los valles orientales del sur de Tarija (Bolivia), del norte de Salta y del este jujeño (Argentina) formaron parte de la llamada frontera oriental del Tawantinsuyu, en donde el Inca movilizó poblaciones con distintos objetivos (Salas, 1945; González, 1982; Lorandi, 1984; Sánchez y Sica, 1990; Presta, 1997; Oliveto y Ventura, 2009; Oliveto, 2011; Ventura y Oliveto, 2014).

En este trabajo nos referiremos específicamente al este de la Cordillera Oriental, el sector de la Serranía de Santa Victoria, en el norte de Salta (Fig. 1). Esta Serranía fue denominada en los siglos XVII y XVIII como "Cordillera de los Chichas", "Cordillera del Perú" o de "Cozquina". También, sus valles orientales son referidos como ubicados en "las cordilleras del Perú, en las vertientes hacia el Chaco" (Lozano, [1733] 1941: 78), ya que allí comenzaba el territorio que, durante esos siglos, fue conocido como Chaco o Chaco Gualamba (Ventura y Oliveto, 2014). 


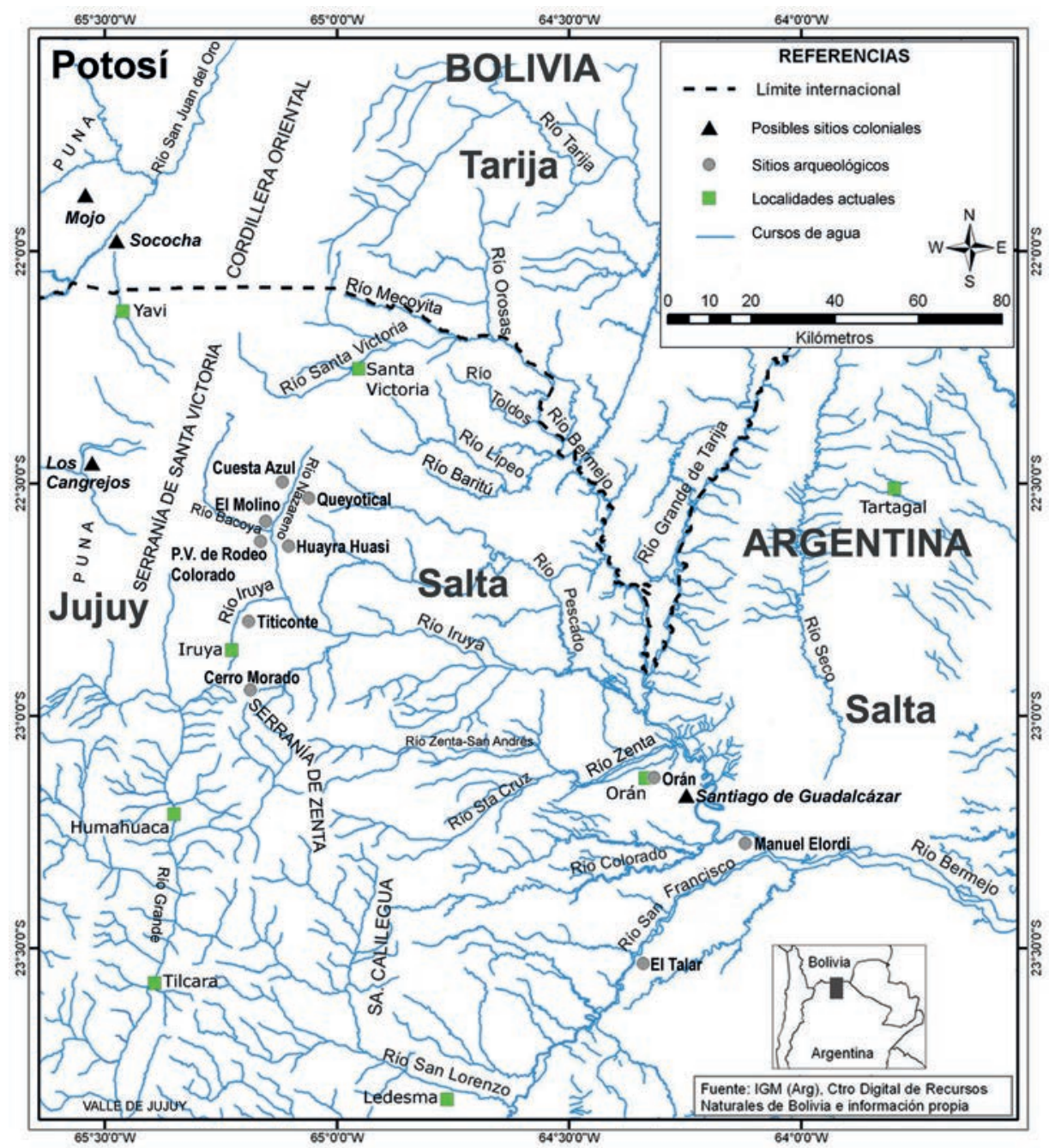

Figura 1. Mapa del sector de los valles orientales del norte de Salta (Argentina) con los sitios mencionados en el texto.

En esos valles orientales el Inca relocalizó diversas poblaciones, entre ellas "los de Titiconde", no identificados étnicamente pero, posiblemente, diferentes a los otros grupos como churumatas, chichas, apatamas y omanatas yapanatas (Salas, 1945) u omanatas y apanatas (Sánchez y Sica, 1990). También, a ciertos grupos jerarquizados, mencionados en la documentación histórica como "orejones" (Lozano, [1733] 1941; Salas, 1945; Iácona y Raffino, 1993; Oliveto y Ventura, 2009; Ventura y Oliveto, 2014; Oliveto y Ventura, 2017).

Consideramos que la ocupación incaica en los valles de Iruya, Nazareno y Bacoya -actuales Deptos de Iruya y Santa Victoria (Fig.1)- se realizó con objetivos principalmente económicos, para lo cual se organizó el espacio conformando un enclave con presencia de poblaciones relocalizadas -mitmaqkuna- y de grupos jerarquizados. Hemos propuesto que una de las principales actividades que se desarrollaron en este sector del oriente de las Serranías de Santa Victoria fueron las minero-metalúrgicas; entre ellas, cierta producción de bienes suntuarios o de prestigio (Ventura y Scambato, 2013). Para cumplir con dichas tareas se organizó un espacio construyendo en los valles un centro administrativo en Titiconte, una extensa andenería agrícola, diversas y complejas estructuras de almacenaje, y un santuario de altura en Cerro Morado a 
1. Radiocarbon Calibration Program 1986-2017 M. Stuiver y P.J. Reimer usado junto con M. Stuiver y P.J. Reimer 1993. Radiocarbon 35:215230. Labcode 001 Description Radiocarbon Age $1030 \quad \pm 120$ Calibration data set shcal $13.14 \mathrm{C}$ Hogg et al. 2013. One Sigma Ranges [start: end] relative area [cal AD 904: cal AD 920]0.0446- [cal AD 968: cal AD 1188] 0.95554. Two Sigma Ranges [start:end] relative area [cal AD 777: ca

Labcode oo1 Description Radiocarbon Age $810 \pm 40$ Calibration data set: shcal $13.14 \mathrm{C}$ Hogg et al. 2013. One Sigma Ranges: [start: end] relative area [cal AD 1228: cal AD 1278] 1. Two Sigma Ranges [start: end] relative area [cal AD 1189: cal AD 1194] 0.006505 -[cal AD 1196: cal AD 1296]0.99395. Ranges marked with $\mathrm{a} *$ are suspect due to impingment on the end of the calibration data set. Hogg et al. 2013.

2. Un brazalete también de aleación cobre-zinc -latón- fue hallado en Doncellas, en la Puna jujeña (Rolandi de Perrot, 1974: 159), con una cronología similar. I
$5.200 \mathrm{msnm}$. Las áreas mineras y pastoriles se ubican también en los sectores altos de la Serranía (Lozano, [1733] 1941; Ventura y Scambato, 2013; Ventura y Oliveto, 2014). Otro sitio con características arquitectónicas incaicas se ha registrado en Queyotical, al este de Cuesta Azul en el valle de Nazareno (Vitry, 2014) (Fig. 1).

En este trabajo, analizaremos ciertos objetos de metal que presentan un alto valor ceremonial y simbólico en la sociedad incaica, relacionados con la minería y la metalurgia. Nos referimos a las chipanas. La palabra "chipana", tanto en quechua como en aymara, significa una "axorca o manilla de los hombres" o "manilla de oro, plata o cobre" (González Holguin, 1608; Bertonio, 1612). Una "manilla" es una pulsera o brazalete que usaban los hombres (Ventura y Scambato, 2013).

Otro objetivo del trabajo es realizar un breve análisis de una categoría de la sociedad inca, los llamados "orejones" en la documentación y en la cartografía de los siglos XVII y XVIII y relacionarlos con su correlato material ya que son mencionados ocupando, en distintos momentos, los valles orientales bajo estudio (Lozano, [1733] 1941; Ventura y Oliveto, 2014; Oliveto y Ventura, 2017). Asociamos a algunos de ellos a ciertos grupos jerárquicos que cumplían funciones relacionadas a los procesos minero-metalúrgicos.

\section{Brazaletes, chipanas y otros ornamentos}

\section{Brazaletes y pulseras en los valles orientales salteños}

La presencia de brazaletes y pulseras de metal ha sido registrada en otros valles orientales del norte de Salta en momentos previos a la ocupación incaica. Formaban parte de acompañamientos funerarios en cementerios (Manuel Elordi 1 y El Talar) y en Orán, sitios localizados en ambiente de Selva pedemontana, ubicados en las cercanías de la unión de los ríos Bermejo y San Francisco (Fig. 1). Dos fechados, en Manuel Elordi 1, los ubican entre ca. 780 y 1300 d.C (1030 \pm 120 AP [Gak 9900] y $810 \pm 40$ AP [UGA 8025]. ${ }^{1}$ Los brazaletes analizados mostraron cobre y zinc -latón- y bronce en su composición (Ventura, 1985; Ventura, 2001). ${ }^{2}$ Junto con estos brazaletes y pulseras se hallaron otras piezas de metal de distintas composiciones -cobre, bronce-, tales como anillos, campanillas, placas rectangulares y representaciones de zoomorfos, entre ellas, una llama de oro-plata-cobre (Palacio y Rodríguez, 1985; Ventura, 1985, 1999; Ventura y Scambato, 2010). Hemos propuesto que algunas de estas piezas de metal han tenido su origen en el altiplano boliviano, llegando a la selva a través de un complejo sistema de intercambio que abarcaba las tierras altas y los valles orientales de Bolivia, la Puna jujeña y la quebrada de Humahuaca (Ventura, 1999).

\section{Minas y chipanas en el Tawantinsuyu}

El conocimiento por parte del Inca de la riqueza metalífera de la Serranía de los Chichas y la fertilidad de estos valles pudo provenir de datos previos, debido a las ocupaciones anteriores que presentan algunos de los sitios arqueológicos estudiados. Sarmiento de Gamboa refiere que Topa Inga Yupanqui:

Tenía mandado que cuando algún mercader trajese a vender algún oro ó plata ó piedras preciosas y otras cosas exquisitas, le echasen mano y le preguntasen de donde lo había habido o sacado, y desta manera daban noticia de las minas 
y lugares donde lo habían sacado; y desta manera descubrió grandísima cantidad de minas de oro y plata y colores muy finos (Sarmiento de Gamboa, [1572] 2012: 431-432).

Este Inca tenía dos gobernadores llamados suyoyoc apo, uno de los cuales residía en Xauxa y el otro en Tiaguanaco. Por Pedro de Cieza de León ([1550] 2005: 224-225) sabemos que en las cabeceras de las provincias el Inca colocaba mayor cantidad de plateros. Un caso corresponde a Xauxa -actual Jauja, ubicada al este de Lima, en el valle del Mantaro, en Perú- en donde "había un gran número de plateros que labraban vasos y vasijas de plata y oro para el servicio de los Ingas y ornamentos del templo" (Cieza de León, [1550] 2005: 225).

En el detallado estudio que Berthelot (1986) realiza sobre la minería incaica destaca que las minas de oro y de plata pertenecían al Inca, al igual que las mejores minas de otros minerales. Y, en el Tawantinsuyu los indígenas:

\begin{abstract}
Aunque no tributaban ni oro ni plata, salvo donde el inga tenía minas, mandaba ir los indios que le parescia, para que le sacasen oro y plata para sus vasos y otras cosas de su servicio; y en la provincia que había plateros, le daban chipanas en poca cantidad, porque una guaranga dicen que daba una chipana; y de ordinario, según dicen, en la provincia que tenía minas o las alcanzaba cerca, pedía el inga para sacar oro dellas de cient indios uno (Santillán, [ca. 1563] 1968: 115-42, el resaltado en cursiva es nuestro).

[...] y el oro y la plata que sacaban de las minas y chipanas y brazaletes, todo esto lo llevaban al Cuzco sin que quedase cosa en poder del curaca, porque no podían tener cosa alguno de ello si no fuese dado por el inga, y el los mandaba a cada provincia la cantidad que habían de sacar de oro y plata por unas pesas que el les daba, que a lo que dicen era poca cantidad (Santillán, [ca. 1563] 1968: 116-51).
\end{abstract}

Destaca también que "en tiempos del inga no le tributaban ni daban oro ni plata más que las dichas chipanas y brazaletes", que era muy poca cantidad. (Santillán, [ca. 1563] 1968: 125-66, el resaltado en cursiva es nuestro).

Según este oidor de la Audiencia de Lima, en el Tawantinsuyu las provincias ricas en minas proporcionaban metales preciosos y cada provincia contribuía con una chipana de estos metales. Al Inca se destinaban los mejores productos y, para cumplir con ello, los curacas locales viajaban al Cusco llevando presentes que eran entregados en las festividades. El Inca recibía las mejores piezas y un buen número de ellas se repartían entre orejones y curacas como modo de afianzar alianzas y lealtades (Rostworowski, 2001).

Los brazaletes -chipanas-, principalmente de oro y plata, estaban incluidos entre los objetos de enorme valoración y simbolismo dentro de la sociedad andina e incaica especialmente, debido al rol que jugaban en las relaciones políticas, abarcando también el ámbito de lo sagrado (Fig.2). 


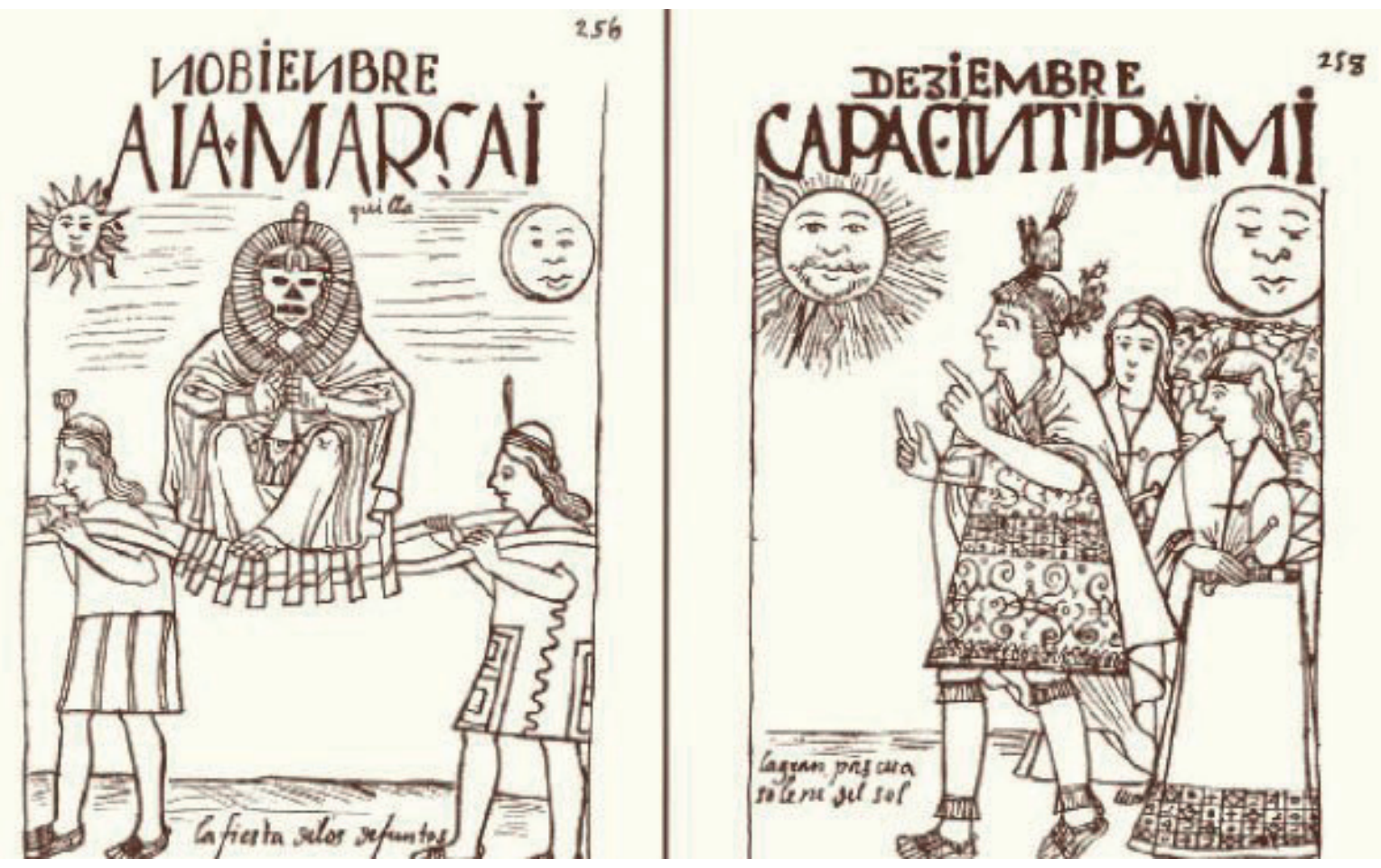

Figura 2. Uso de chipanas entre los emblemas de poder usados en festividades y celebraciones (Guaman Poma [1615] 1980: 258-260).

Brazaletes de oro y de plata se hallaron en los brazos de niños -varones- sacrificados en las capacochas en diversos lugares del Tawantinsuyu, entre ellos, en el cerro El Plomo y en el Llullaillaco (Reinhard y Ceruti, 2010), éste último en Salta, o entre las miniaturas que acompañaban esos sacrificios. También, en sitios ceremoniales en el centro del imperio, en Choquepukio (Andrushkoet al., 2011) o en el Coricancha (Barreda Murillo, s/f) .

Richard Burger (2004) refiere que el único objeto de oro registrado en Machu Picchu es un brazalete tubular dejado como ofrenda antes de colocar los gruesos cimientos de piedra que permitió la construcción de ese sitio y la estabilidad que hoy tienen sus construcciones.

Como ya mencionamos, las chipanas eran parte de los adornos que llevaban los nobles incas y se contaban entre los objetos suntuarios distribuidos por el Inca como obsequios diplomáticos (Ceruti, 2003). Estas chipanas, al igual que los textiles y el calzado (uxutas), participaban de la condición sagrada que les daba el hecho de "ser del Inka" (Martínez, 1994: 31). Todos los bienes que se repartían en esas ceremonias se consideran del Inca. Cieza de León ([1550] 2005: 348) refiere que los servicios de los orejones eran recompensados con la entrega de ropa de lana, plumas, brazaletes de oro y plata y con mujeres.

Helena Horta Tricallotis (2008), quien analiza ciertas insignias de jerarquía de la sociedad inca-canipus y tincurpas-, registra en la documentación a las chipanas o brazaletes entre los adornos de metal usados por varones nobles, tanto vivos como muertos - "bultos" de antepasados- que seguían participando de la vida social y política del Cusco.

Luis Barreda Murillo les atribuye una extraña función a estas piezas al considerar que las chipanas son "formas cónicas bien pulidas que sirvieron para concentrar los rayos del sol y producir fuego con ayuda de fibras de algodón, 
alpaca o llama, untadas con sebo de camélidos". Además, refiere que en el caso de estas piezas realizadas en bronce, al ser tratadas químicamente recobran su brillo original, y destaca que ese brillo supera al de los objetos de oro. Posiblemente esa fue una característica por la cual los incas tuvieron gran preferencia por los objetos de bronce (Barreda Murillo, s/f). Sabemos que el brillo fue una condición buscada en los objetos durante el incario y que los adornos con superficies brillantes eran parte de la parafernalia ritual de las personas de estatus privilegiado. Morris y von Hagen (2011: 63) ${ }^{3}$ señalan que estas piezas con brillo no sólo incrementaban el espectáculo de la ceremonia, sino que su uso por parte de personas importantes y poderosas realzaba el valor de los objetos y el estatus de quienes los usaban.

\section{Orejones en los valles orientales}

El estado Inca sostenía grupos de artesanos o especialistas cuyo trabajo mantenía una calidad y un estilo distintivo, que lo caracterizaba. Tanto desde la etnohistoria como desde la arqueología se ha establecido la especialización de ciertos grupos de artesanos en cerámica, textiles, lapidaria y metalurgia, quienes realizaban dichas tareas en distintos centros distribuidos a lo largo del Tawantinsuyu, a fin de satisfacer las demandas estatales.

Santillan ([ca. 1563] 1968: 125-66) refiere que en el Tawantinsuyu eran mineros y "oficiales de aquel oficio" quienes tenían minas en su territorio. Mientras que quienes supervisaban las minas del Inca eran representantes del poder central que podían ser nobles de linaje inca (Berthelot, 1986).

Los llamados "orejones" fueron poblaciones jerarquizadas que cumplieron funciones organizativas, de control y mando en los enclaves incaicos dentro de los espacios sometidos (Pärssinen, 2003; Someda, 2004). Hemos analizado las menciones que la documentación y la cartografía del siglo XVII y XVIII registran sobre la categoría incaica de "orejones" en relación a ciertos grupos jerarquizados que ocuparon los valles orientales bajo estudio (Ventura y Oliveto, 2014; Oliveto y Ventura, 2017). La referencia más antigua que se conoce sobre ellos en estos valles orientales se halla en una carta geográfica de la provincia jesuítica del Paraguay -jurisdicción a la que pertenecía el norte de Salta- que elaboró en 1609 Diego de Torres ([1609] 1927). Allí, localizados junto a un afluente del Bermejo, en unas serranías sin identificación, se hallan los "Orechones" a los que se indica como indios infieles. Consideramos que esta carta podría presentar una imagen cercana a momentos prehispánicos de estos valles (Ventura y Oliveto, 2014).

Otra referencia corresponde al padre Gaspar Osorio quien, en una carta de 1628, se refería a la ubicación de los churumatas a unas dos jornadas de la nueva población de Santiago de Guadalcázar, situada en la selva pedemontana -de la actual Salta- (Fig. 1), y que "junto a estos Churumatas están los Orejones, indios que hablan la lengua aymará, que es una de las generales que corren en el Perú" (Lozano, [1733] 1941: 164).

En un Memorial que Martín de Ledesma Valderrama escribe en 1631, en su huida desde la destruida ciudad de Santiago de Guadalcázar, menciona a "dos naciones de indios naturales del reino del Perú" ocupando los valles al oriente de la Cordillera de los Chichas, una es de "indios ingas del Cusco capitanes del Inga" y la otra es de churumatas, y ambas son "gente rica de plata y minerales" (Oliveto y Ventura, 2017: 266-276).
3. La traducción es nuestra. 
4. Un pillo o pillu es una corona de oro u otro metal (González Holguin, 1608).
También, en una carta de 1644 Ledesma Valderrama refiere que en estos valles "hay 3.000 indios de los ingas orejones del Cuzco y churumatas" y que "muchos de estos indios son vecinos a los minerales e ingenios de los chichas y lipez y a la villa de Potosí" (Pastells, 1915: 94).

Posteriormente, en la primera mitad del siglo XVIII, el jesuita Pedro Lozano utilizando esa información -aunque sin citarla explícitamente- y otra documentación de esa etapa conquistadora del Chaco, durante la primera mitad del siglo anterior, alude a churumatas y a chichas orejones. El jesuita agrega a los chichas, a quienes no menciona Ledesma Valderrama, y le atribuye la categoría de "orejones" (Oliveto y Ventura, 2017). Lozano refiere a dos tipos de poblaciones que ocupaban el Chaco, unas "que iban a recoger los tributos para el Inca, y las otras [...] eran de varias naciones del Perú que labraban algunas alhajas de plata, al modo de los plateros sacándola de minerales" (Lozano, [1733] 1941: 18).

En su relato destaca que:

Y era fama y tradición constante, que de aquella cordillera que corre de norte a sur, y se divisaba desde la ciudad de Guadalcázar, sacaban los Orejones del Cuzco grandes cantidades de oro y plata, que contribuían sus moradores para llevar a aquella corte en tiempos pasados, para presentar a los emperadores ingas (Lozano, [1733] 1941: 20).

Refiere, también, que los churumatas y chichas orejones:

están metidos en un valle que hacen las cordilleras del Perú en las vertientes del Chaco [...] Dicen que serán como seis mil almas. Andan vestidos como en el Perú, de lana de los carneros de la tierra que tienen, y que labran minas de plata, de cuyo metal forman su ajuar, y hacen adornos para sus mujeres, y los hombres chipanas, penachos y pillos ${ }^{4}$ para bailar al uso del Inga. Los chichas orejones, que viven en dichos valles junto con los churumatas son indios que ocupaban los emperadores ingas en las minas y conquista de la cordillera, los cuales cuando supieron la entrada de los españoles en el Perú y la muerte que habían dado al Inga Atahualpa en Cajamarca, y que se habían apoderado del Cuzco, no quisieron volver al Perú y se quedaron en tierra de los churumatas (Lozano, [1733] 1941: 78).

\section{Sumando otros registros}

Como ya mencionamos, la Cordillera del Perú o Cordillera de los Chichas corresponde a la Serranía de Santa Victoria -Cordillera Oriental-, la cual presenta variedad de yacimientos metalíferos. Las manifestaciones de plomoplata-zinc son frecuentes, así como las de cobre, níquel, hierro y oro. En el norte se presentan también placeres auríferos (Rubiolo, 2003; Ventura y Scambato, 2013). Es decir que la base geológica de la zona sustenta la posibilidad de la explotación minero-metalúrgica que manifiestan las fuentes documentales y el registro arqueológico que presentamos a continuación.

El hallazgo de piezas de metal consideradas de prestigio se ha registrado en varios sitios arqueológicos de los valles orientales del norte de Salta. Específicamente, en los asentamientos de los valles Nazareno, Bacoya e Iruya en el sector de Pastizales de Neblina (3000-3500 msnm), ubicados al este de las Serranías de Santa Victoria (Fig. 1). 
En las excavaciones llevadas a cabo por Márquez Miranda en estos valles, entre 1934 y 1938, se hallaron 63 objetos de metal. ${ }^{5}$ De ellos, 36 provienen del sitio Pueblo Viejo de Rodeo Colorado (PVRC) (Fig. 1), en donde se registraron diversas piezas para usar en brazos y manos. Entre ellas, tres brazaletes tubulares, tres pulseras de distintos anchos, dos brazales, tres manoplas y anillos (Fig. 3). Además, otras piezas provenientes de este asentamiento residencial corresponden a elementos de prestigio, como una placa circular de plata -tincurpa- y otras placas circulares de plata de menor tamaño. En este sitio se hallaron también numerosas cuentas de collares realizadas en turquesa, sodalita, material malacológico y en tobas volcánicas locales, ${ }^{6}$ registrándose, además, instrumentos musicales y textiles de muy buena calidad (Márquez Miranda, 1939; Ventura, 2016).

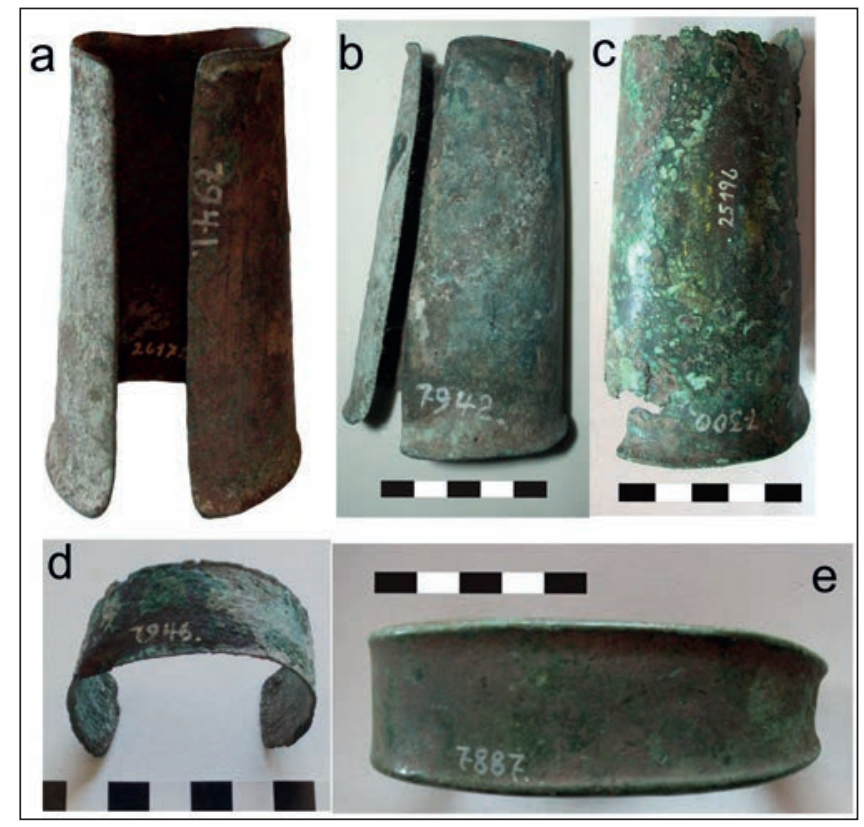

Figura 3. a-b-c) brazaletes tubulares; d) pulsera; e) manopla de Pueblo Viejo de Rodeo Colorado (a: 7941-26172; b: 7942-26173; c: 7300-25196; d: 7946-26177; e: 7887-26115). Corresponde a la numeración del Inventario del MCNLP, Colección Márquez Miranda.

De Cuesta Azul (Fig. 1) proviene una pulsera de plata con decoración grabada de triángulos con puntos en su interior (Márquez Miranda, 1939; Ventura y Scambato, 2013). Mientras que en Huayra Huasi, otro asentamiento en el valle de Nazareno, Márquez Miranda (1939) registró cuatro pequeñas piezas de oro y una placa rectangular de bronce de las denominadas "placas santamarianas" tardías, de amplia distribución en momentos incaicos.

\section{Análisis}

Como resultado de los análisis llevados a cabo sobre 35 piezas de metal de estos valles orientales del norte de Salta hemos constatado la presencia de objetos de oro, plata, bronce, cobre y una bola de plomo (Ventura y Scambato, 2013). Diez de estas piezas son pulseras, brazaletes tubulares, brazales y manoplas, de variada composición. Mencionamos ya una pulsera de plata, otras seis son de bronce estañífero, mientras que dos brazaletes tubulares y una pulsera presentan níquel en su composición -cuproniquel- (Tabla 1).
5. Estos materiales forman parte de la Colección Márquez Miranda, depositada en el Museo de Ciencias Naturales de La Plata (MCNLP).

6. En todos los casos se llevaron a cabo los análisis para la determinación de dichas materias primas. 
La aleación cobre-níquel en estas piezas de metal analizadas provenientes del sitio Pueblo Viejo de Rodeo Colorado (PVRC) (Ventura y Scambato, 2013; Ventura et al., 2016) nos ha llevado a proponer que la fabricación de estos objetos sería regional, pues considerando que "los minerales de níquel son muy escasos en la zona andina" (Lechtman y Macfarlane, 2006: 511) y el área de estudio tiene ricas fuentes de este mineral, la propuesta merece ser analizada (Ventura et al., 2016).

\begin{tabular}{|c|c|c|c|c|c|c|c|c|c|c|c|}
\hline $\begin{array}{l}\text { Invent. } \\
\text { MCNLP }\end{array}$ & Categor. & Proced. & $\begin{array}{l}\text { Peso } \\
\text { (grs) }\end{array}$ & Metal & $\mathrm{Cu}$ & Sn & $\mathrm{Zn}$ & $\mathrm{Ag}$ & $\mathrm{Ni}$ & $\mathrm{Pb}$ & $\mathrm{Fe}$ \\
\hline 25342 & Pulsera & Cuesta Azul & 20 & Plata & 5,1 & & & 94,9 & & & \\
\hline 26261 & Manopla & Iruya-Santa Victoria & 92,4 & Bronce & 87,54 & 12,46 & & & & & \\
\hline 25197 & Brazal & PVRC & 140,9 & Bronce & 94,39 & 5,6 & & & & & \\
\hline 26166 & Pulsera & PVRC & 9,1 & Cupro-Níquel & 96,18 & & & & 3,85 & & \\
\hline 26172 & Brazalete tubular & PVRC & 88,6 & Cupro-Níquel & 94,22 & & & & 4,36 & & \\
\hline 26173 & Brazalete tubular & PVRC & & Cupro-Níquel & 90,72 & & & & 3,35 & & 2,3 \\
\hline 26115 & Manopla & PVRC & 170,9 & Bronce & 97,01 & 2,86 & & & & & \\
\hline 26185 & Brazal & PVRC & 109,2 & Bronce & 90,16 & 5,31 & & & & & \\
\hline 25196 & Brazalete tubular & PVRC & 40,2 & Bronce & 94,5 & 5,5 & & & & & \\
\hline 26116 & Pulsera & PVRC & & Bronce & 88,29 & 11,27 & & & & & 0,5 \\
\hline Anális.Balmás & Pulsera & El Molino & & & & 10,3 & 0,31 & & & 6,7 & \\
\hline Anális.Balmás & Pulsera & Cuesta Azul & & & & 13,2 & 0,44 & & & 10,1 & \\
\hline
\end{tabular}

Tabla 1. Resultados de los análisis de composición de los brazaletes tubulares, pulseras, manoplas y brazales de los sitios arqueológicos del valles de Nazareno. Corresponde a la numeración del Inventario del MCNLP: Colección Márquez Miranda y los realizados por Juan Carlos Balmás. (PVRC significa Pueblo Viejo de Rodeo Colorado).

\section{Chipanas en el siglo XVI}

Con la entrada española al Tawantinsuyu ciertos objetos de metal mantuvieron su importancia. Para los hispanos, por su valor debido a los materiales en que estaban confeccionados y para los indígenas por su condición de elementos de prestigio o por su valor simbólico.

7. Un socorro consistía en “Dinero, alimento u otra cosa con que se socorre" o "Ayudar o favorecer en un peligro o necesidad", según el Diccionario de la Lengua Española (1936).

8. Agradezco este dato a la Dra. Lydia Fossa.
Las chipanas aparecen registradas como joyas de metales preciosos -oro y platautilizados como socorros. ${ }^{7}$ Un ejemplo temprano es registrado en declaraciones de los wanca, que datan de 1533 y 1534, hechas en $1558 .^{8}$

Primeramente, luego que en el dicho repartimiento de Atunxauxa supimos que el dicho marqués había entrado en Caxamalca e muerto a Atavalipa, fue Manco Cusichac [...] al dicho valle de Caxamalca juntamente con el dicho don Diego Ynaupari cacique del dicho repartimiento e le llevaron de socorro al dicho marques con sus yndios que fueron dozientos e sesenta e un un yndios cargados de mays e papas e quinoa e trezientos e un pesos de oro en chipanas e cocos e otras joyas e trezientos e un pesos en plata e asymesmo en mates e chipanas e topos e otras cosas que los valieron y veinte carneros de la tierra e quarenta pieças de ropa de cumbi muy ricas; sesenta yndios para su servicio del dicho marques e sus soldados e asymesmo los dichos doszientos e setenta y un yndios que fueron cargados con el dicho socorro quedaron en el dicho valle de Caxamarca por yanaconas de los soldados del dicho marques que ninguno dellos bolvio al dicho repartimiento de Atunxauxa [...]. Todo lo cual llevaron al dicho marques a Caxamarca e lo rescibieron por amigo (Pärsinnen y Kiriharju, 2004: 197) (el resaltado en cursiva es nuestro).

Durante el siglo XVI, y con el objetivo de afirmar sus jerarquías de autoridad, los ajuares de algunos caciques registran numerosos bienes tradicionales 
andinos; entre ellos, las mencionadas chipanas. Mercedes del Río, (2010) refiere que en los inventarios de cuatro caciques de Pocona y Totora en la provincia de Charcas había cantidad de objetos de oro y plata y que el adorno más difundido eran las chipanas o brazaletes. Los cuatro tenían chipanas de oro y de plata. Pedro Arapa (Pocona) tenía cinco de oro. Los otros tres de Totora tenían una sola de oro cada uno, pero todos tenían chipanas de plata, algunos caciques tenían dos, uno de los cuales era un brazalete mitad de oro y mitad de plata (del Río, 2010: 214, tabla 4).

Otros elementos tradicionales de prestigio que registra esta investigadora en los inventarios de los caciques son los collares de turquesillas y piedras verdes y de cuentas azules, pillos -coronas- de oro y de plata, así como también collares de turquesas con plumas de juríes y coronas de plumas de guacamayos. Del Río refiere que "los plateros de Sipesipe" eran mitmaqkuna Ica yungas, que seguían llamándose así hacia 1570, que proveían objetos de oro y plata a los caciques locales, o por lo menos a los de Pocona y de Tapacarí (del Río, 2010: 208).

Desde los inicios de la conquista española los conocimientos de los especialistas metalurgistas indígenas fueron utilizados para sus propios beneficios por sus encomenderos y, en algunos casos, de forma fraudulenta. Un ejemplo temprano, y en relación a las pulseras o manillas, es registrado en Colombia en 1555. Un documento detalla el caso de alteración aurífera en las pulseras que fabricaba el grupo de Zimpieguas, cuyo tributo mensual para el encomendero era de quince manillas, las que eran producidas por un cacique y sus capitanes $^{9}$ (Martínez Garnica, 1989). También en este caso las pulseras eran el elemento de intercambio y de prestigio, elaboradas por un grupo jerárquico de la población indígena.

La palabra "chipana", además de brazalete de hombre, significa "gala de barones", "el que da honor y orgullo a su pueblo", aunque también podría referir a la especialización de un grupo de orfebres (Ventura y Scambato, 2013).

El Juicio de residencia del Dr. Gabriel de Rearte de 1575 (AGI, Justicia 463, Escribanía 498 A), que está trabajando la Dra. Lydia Fossa, registra al menos quince apariciones de indígenas de apellido Chipana, son mitayos que provienen de la zona de Jauja, afectados por la minería del azogue en Huancavelica -por ejemplo, "del ayllu Cayana, curaca don Diego Chipana". ${ }^{10}$

Analizando documentación de un siglo después, Luisa Vetter Parodi (2013) detecta en Xauxa a media docena de indios plateros de apellido Chipana, del ayllu plateros, entre 1664-1682 (Tabla 2).
9. Martínez Garnica (1989) analiza el documento "Averiguaciones sobre el modo como los indios del pueblo de Zimpieguas fabricaban manillas del oro. Tamalameque, del 15 de enero de 1555 " (AGl, Justicia 587-A. Ff 739r-743 v).

10. Agradecemos estos datos de la Dra. Lydia Fossa (junio, 2017).

\begin{tabular}{|l|l|l|l|l|l|l|l|}
\hline \multicolumn{1}{|c|}{ Nombre } & Ocupación & \multicolumn{1}{|c|}{ Años } & Repartimiento & Provincia & Doctrina & Ayllu & Archivo \\
\hline Ignacio Chipana & Platero & $1664-1682$ & Hananguanca & Xauxa & Huancayo & Plateros & ARH,ff 707-718 \\
\hline Juan Chipana & Platero & $1664-1682$ & Hananguanca & Xauxa & Huancayo & Plateros & ARH,ff 707-718 \\
\hline Lorenzo Chipana & Platero & $1664-1682$ & Hananguanca & Xauxa & Huancayo & Plateros & ARH,ff 707-718 \\
\hline Martín Chipana & Platero & $1664-1682$ & Hananguanca & Xauxa & Huancayo & Plateros & ARH,ff 707-718 \\
\hline Sacarías Chipana & Platero & $1664-1682$ & Hananguanca & Xauxa & Huancayo & Plateros & ARH,ff 707-718 \\
\hline Pedro Chipana & Platero & $1664-1682$ & Hananguanca & Xauxa & Chupaca & Plateros & ARH,ff 707-718 \\
\hline
\end{tabular}

Tabla 2. Plateros indios de Jauja en los siglos XVI y XVII (Vetter Parodi, 2013. Anexo 2: 352). 
En las visitas de 1645 y 1659 a la jurisdicción de Tarija, analizadas por Carlos Zanolli (2008), se registran también a varios indios de apellido Chipana. Sin embargo, en esa documentación ellos no están relacionados con alguna actividad específica. El único platero mencionado es un indio llamado Alonso de edad de 80 años natural del Cuzco (Zanolli, 2008: 112). Este investigador, también refiere que los chichas entregaban tributo a los chiriguanos en ropa de cumbe, pillos, manillas de plata y azuelas de hierro, entre otras cosas.

\section{El Chaco colonial}

Los valles del oriente de la Cordillera de los Chichas, que formaron parte de la frontera oriental del Tawantinsuyu, pasaron a integrar el llamado Chaco o Chaco Gualamba durante la colonia. Aunque la ocupación hispana fue muy tardía -segunda mitad del siglo XVIII-, en ciertos casos, como en Titiconte, en el valle de Iruya y, más al sur, en el valle de Zenta-San Andrés, parte de las poblaciones indígenas fueron tempranamente retiradas y relocalizadas en las cercanías de localidades bajo el dominio español (Ventura y Oliveto, 2014).

En un mapa de autor anónimo, realizado posiblemente en la primera mitad del siglo XVII -depositado en la Biblioteca Nacional de Francia, en París- (Saignes, 1985) se señalan asentamientos indígenas y españoles, centros mineros y cerros sagrados en la Provincia de Charcas. Esta carta incluye los valles ubicados al sur de Tarija. Allí, al oriente de unas serranías, se lee "A las faldas de esta cordillera que divide el Chaco del Piru estan los churumatas chichas y orejones hay minas de plata". Y se señala a "Los chipanas", representados con unos círculos concéntricos, signos cartográficos que podrían señalar a poblaciones indígenas no reducidas (Ventura y Oliveto, 2014) (Fig. 4).

Ya hemos propuesto que la mención de "Los chipanas" pudo haber nombrado a una población caracterizada por realizar tareas de orfebrería conocida por la fabricación de pulseras, brazaletes, coronas y otros objetos de metal (Ventura y Scambato, 2013). También nos hemos preguntado si esta denominación podría asociarse a "los plateros" que menciona Lozano ([1733] 1941: 78-79) quien, como ya citamos, refiere que los churumatas y chichas orejones, además de explotar las minas de plata, utilizaban ese metal para fabricar chipanas para los hombres, para lucir en festividades a la antigua usanza incaica.

Consideramos que en estos valles pudo haber, como en otros sectores del Tawantinsuyu, poblaciones de mineros y de artesanos plateros, y que estas poblaciones, llevadas por el Inca, podrían ser "Los chipanas" destacados en este mapa del siglo XVII, mencionados por los españoles y ubicados en un territorio aún no ocupado por ellos.

En 1630, Lucas Rendón daba cuenta de que los mineros de la Villa de Potosí conocían las riquezas de la cordillera que alojaba a estas poblaciones indígenas, y que deseaban catar sus minerales, pero no lo hacían por temor a los ataques de los indios del Chaco y de otros indios de guerra que habitaban esos valles (Lozano, [1733] 1941: 20)

La posterior entrada hispana en estos valles orientales pudo causar importantes cambios sociales y en la tecnología minero-metalúrgica indígena. El uso del plomo en las aleaciones parece haberse utilizado y se registra en dos pulseras. Los análisis realizados por Juan Carlos Balmás (1999) en siete piezas de metal; entre ellas, dos pulseras, una de El Molino y otra de Cuesta Azul 


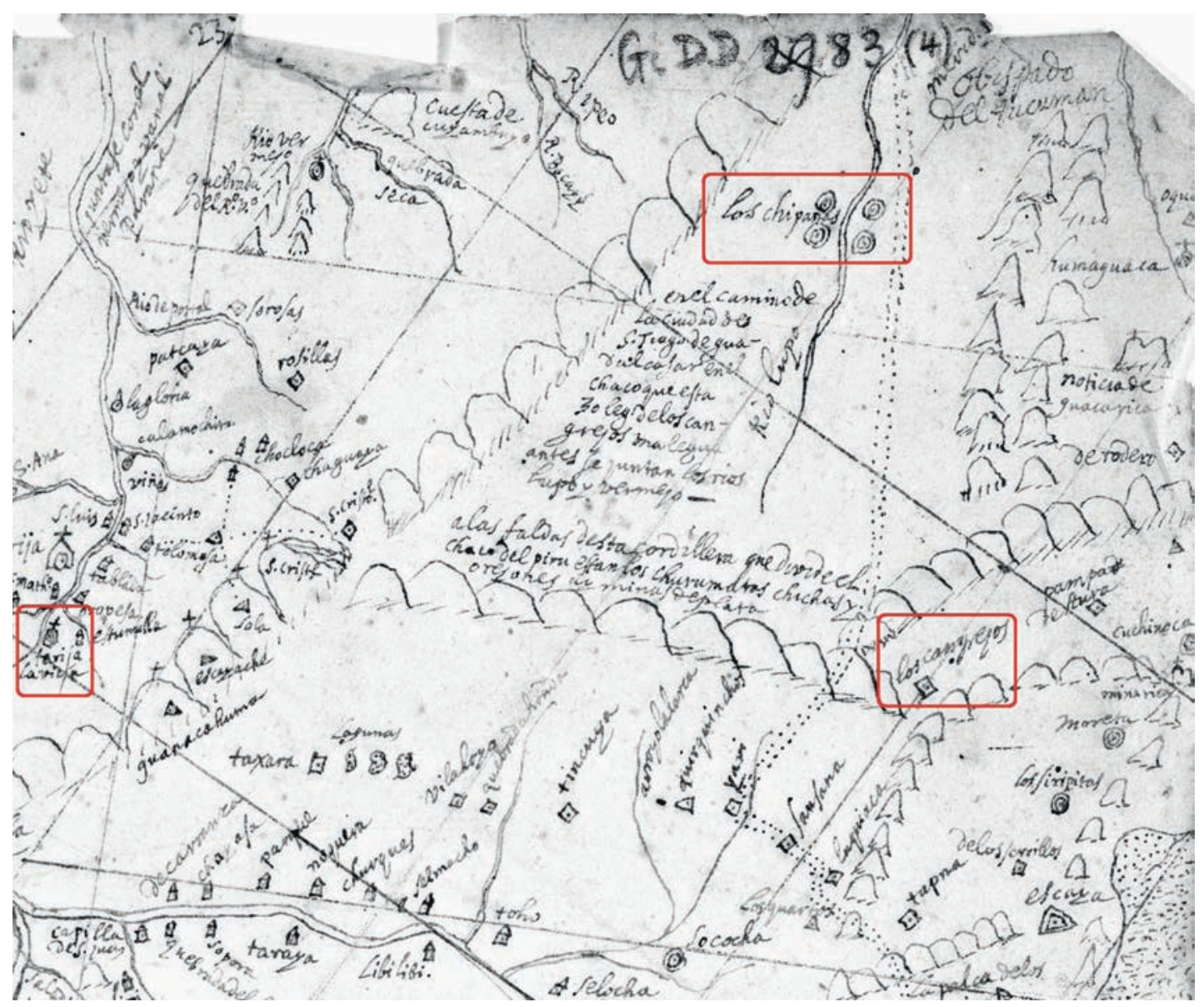

Figura 4. Sector del mapa anónimo de la Provincia de Charcas depositado en la Biblioteca Nacional de París, donde se señala a Los Chipanas. Se ha marcado también la ubicación de Tarija y Los Cangrejos.

(Fig. 1), muestran no sólo la fabricación menos esmerada de estas piezas sino, también, el uso de la aleación cobre-plomo (Tabla 1).

\section{Consideraciones finales}

Algunos objetos de metal integraron un conjunto de piezas que compartieron una antigua y amplia tradición panandina, entre los que se cuentan las pulseras y los brazaletes. La presencia de estas piezas de metal ha sido registrada en diversos sitios arqueológicos del Noroeste argentino (NOA); entre ellos, en los cementerios de la selva pedemontana, en el norte de Salta, en momentos previos a la llegada incaica. Los brazaletes analizados mostraron cobre y zinc -latón- en su composición (Ventura, 1985). Sin embargo, en esos sitios no se detectaron ciertas formas que caracterizan al conjunto de objetos de metal del valle de Nazareno hallados, principalmente, en Pueblo Viejo de Rodeo Colorado. Nos referimos a las placas circulares de plata, las manoplas, los brazales y la placa rectangular de bronce "santamariana" de Huayra Huasi, piezas que, posiblemente, puedan brindarnos una idea aproximada de su ubicación temporal.

Ciertos objetos de metal fueron elaborados y distribuidos por distintas sociedades en momentos previos a la ocupación incaica y continuaron en uso bajo el poder estatal. Algunas de estas piezas tuvieron mayor aceptación que otras por 
11. La traducción es nuestra.

12. Owen (2012: 180) registra la mayor concentración de estas placas en Moquegua, Perú. parte de incario, lo que permitió, o no, su dispersión hacia las áreas centrales del imperio. Se ha planteado que una de las modificaciones implementadas en el NOA por los incas y en la metalurgia fue la movilización de productos terminados o semi-terminados a otras áreas del imperio (González y Gluzman, 2007). Las denominadas "placas santamarianas" rectangulares de bronce, tardías, muestran una amplia distribución en momentos incaicos. Con origen en los valles Calchaquíes, han sido registradas en la Puna jujeña, en Catarpe en el norte de Chile, en los valles de Bolivia, en el sur de Perú (Moquegua), llegando a Sacsawaman en el corazón del Imperio (González, 1992; Cruz, 2009-2011).

Las manoplas son otras piezas con origen en el NOA que se han registrado en Pueblo Viejo de Rodeo Colorado. Alberto R. González y Víctor Núñez Regueiro (1968-1969) consideran que corresponden a momentos tardíos e incaicos, perdurando hasta la llegada de los españoles, aproximadamente entre el 1200 y el 1550 d. C. También, Eugen Mayer (1986: 49) atribuye a las manoplas una cronología tardía y, en algún caso (La Paya), las ubica dentro de un contexto incaico. Tres de estas piezas se han detectado en PVRC y en otros sitios arqueológicos del NOA, principalmente en la Puna jujeña, la Quebrada de Humahuaca y, en el norte de Chile, en Calama, Antofagasta y Quillagua (González y Núñez Regueiro, 1968-1969), pero no parecen haber llegado a Bolivia ni a Perú.

En relación a los brazales, Owen (2012: 99) considera que tuvieron una dispersión limitada y que no se han registrado en Perú debido, posiblemente, a que "pudieron haber quedado fuera de uso antes que el Inca llegara a la región, o pudieron haber sido parte de la tecnología militar o iconografía local, que el Inca pudo no haber tenido interés en difundir". ${ }^{11}$

Otras piezas de metal debieron su dispersión a la expansión incaica, dado los movimientos de poblaciones, de objetos y de conceptos simbólico-religiosos y jerárquicos llevados a cabo a través de un sistema de transmisión estatal, por el que se distribuyeron a la largo del Tawantinsuyu. Podríamos considerar en este caso a las placas circulares de plata de mayor tamaño que corresponderían a las denominadas tincurpas, insignias que usaban los nobles incas en ciertas festividades (Horta Tricallotis, 2008: 85). ${ }^{12}$

Algunos objetos de oro y de plata pudieron haber tenido un origen en diversos centros del Tawantinsuyu y su distribución se llevó a cabo dentro del sistema estatal. Por ejemplo, las piezas de oro -pequeñas campanillas o cubiletes- y de plata -tincurpas y placas circulares de menor tamaño- que se registran en los asentamientos de estos valles. Estas piezas tuvieron una amplia distribución y podrían ser relacionadas con grupos jerarquizados, "orejones", que cumplirían ciertas funciones de control y administración en las producciones desarrolladas en estas serranías y valles orientales. También podríamos incluir a la pulsera de plata de Cuesta Azul cuya decoración, grabada con triángulos con puntos en su interior, es similar a la de una pulsera de bronce y plata hallada en Machu Picchu (Burger y Salazar, 2004: 180), y a la decoración de una pinza del valle del Mantaro, en el Perú. En este último caso Costin et al. (1989: 125) consideran que este tipo de decoración grabada es una innovación inca en la metalurgia wanka. Además, en las cercanías de Jauja fueron hallados fragmentos de cintas de metal con motivos decorativos similares (Owen, 2001).

Sin duda, las chipanas, brazaletes y pulseras tuvieron un notable valor simbólico, tanto para que el Inca exigiera su entrega a las provincias con minas, al darle valor de objetos sagrados e incluirlos en las capacochas y en otras ofrendas de alto significado ritual, como hemos detallado. También eran recibidas por 
el Inca y distribuidas en el sistema de regalos y contra-regalos a curacas y orejones, siendo ornamentos masculinos y de grupos jerarquizados.

La determinación de la composición de estas piezas y el conocimiento de la geología regional es relevante para contemplar la posibilidad de fabricación local, tal como podrían ser considerados los brazaletes y pulseras de cobreníquel, lo cual aún debe ser verificado con mayores análisis.

Consideramos que estos valles fueron parte de un sistema macro-regional de producción minero-metalúrgico incaico en el que ciertos grupos jerarquizados se movían entre distintas regiones, movilizando elementos de prestigio. Sin embargo, es muy difícil probar, aún utilizando documentación histórica y arqueológica, la proveniencia o el recorrido de estos grupos especializados. A pesar de haber señalado ciertas relaciones con la zona de Jauja, en Perú, es difícil determinar si los artesanos metalurgistas pudieron haber tenido ese origen y si grupos de esos plateros Chipana fueron trasladados a los valles de Tarija y de Salta durante la ocupación incaica.

Durante la colonización española, las chipanas y otros ornamentos de metal posiblemente continuaron manteniendo su valor como elementos de prestigio y de intercambio entre la población indígena de esos valles orientales. Sin embargo, al destruirse allí el sistema de producción minero-metalúrgico incaico, su fabricación, que pudo haberse reducido al ámbito doméstico, parece declinar en calidad e incorporar tecnología hispana, con el agregado de plomo.

\section{Agradecimientos}

A Lía Guillermina Oliveto, Lydia Fossa, María Ester Albeck, María Florencia Becerra, Federico García Blaya, Luis Borrero, Olga y James Brennan. 


\section{Q Bibliografía}

"Alconini, S. (2004). The southeastern Inka Frontier against the Chiriguanos: Structure and dynamics of the Inka Imperial Borderlands. Latin American Antiquity 15 (4): 389-418.

"Andrushko, V.; Buzon, M.; Gribaja, A.; McEwan, G.; Simonetti, A. y R. Creaser (2011). Investigating a child sacrifice event from the Inca heartland. Journal of Archaeological Science 38 (2):323-333.

»Anónimo (s/f). Mapa Biblioteca Nacional de Francia (BNF). París, Colección Klaproth, Richelieu-Cartes et plans-magasin-GE-2983 (4).

» Barreda Murillo, L. (s/f). Bronces del Qorikancha. Disponible en Internet: http://www. luisbarredamurillo.galeon.com. Consultado el: 21 de mayo 2018.

» Balmás, J. C. (1999). Tradición metalúrgica como componente de identidad cultural: comentarios sobre hallazgos en sitios del pueblo de Tilcara y de los valles orientales de la Cordillera de Santa Victoria. Libro de Resúmenes del XIII Congreso Nacional de Arqueología Argentina 68. Córdoba, Editorial Brujas.

" Berthelot, J. (1986). "The extraction of precious metals at the time of the Inka" en Murra, J.; Wachtel, N. y J-J Ravel (eds.), Anthropological History of Andean Polities: 69-88. Nueva York, Cambridge University Press.

» Bertonio, L. (1612) Diccionario castellano aymara/ aymara castellano [online] Disponible en Internet: www.lenguandina.org/index_aymara.php. Consultado el 15 de octubre de 2011.

» Bronk Ramsey C. (2009). Bayesian analysis of Radiocarbon dates. Radiocarbon 51 (1): 337-360.

" Burger, R. (2004). "Scientific Insights into Daily Life al Machu Picchu" en Burger, R. y L. Salazar (eds.), Machu Picchu, Unveiling the mystery of the Incas. 85-106. New Haven Ct, Yale University.

» Burger, R. y L. Salazar (2004) (eds.). Machu Picchu, Unveiling the mystery of the Incas. New Haven Ct, Yale University.

"Ceruti, M. C. (2003). Llullaillaco. Sacrificios y ofrendas en un santuario inca de alta montaña. Salta, Ediciones Universidad Católica de Salta.

" Cieza de León, P. ([1550] 2005). Crónica del Perú. El Señorío de los Incas. Primera parte. Caracas, Biblioteca Ayacucho.

» Costin, C.; Earle, T.; Owen, B. y G. Russell (1989). “Impact of Inka Conquest on Local Technology in the Upper Mantaro Valley, Peru" en van der Leeuw, S. y R. Torrance (eds.) What's new?: A closer look at the Process of Innovation. One World Archaeology Series 14: 107-139, Londres, Unwin y Allen.

»Cruz, P. (2009-2011). El brillo del señor sonriente. Miradas alternativas sobre las placas metálicas surandinas. Mundo de Antes 6-7: 97-131.

»Cruz, P. e I. Guillot (2010). Terra argéntea. Los reinos del metal prehispánicos en el cruce de la Historia y la Arqueología. Surandino Monográfico 1 (2): 1-26. Universidad de Buenos Aires, Buenos Aires. http://www.filo.uba.ar/contenidos/investigación/ institutos/ravignani/prohal/SM_004_ INFORMES_DE_INVESTIGACION/informes.html. Consultado el: 22 de mayo de 2017.

»Del Río, M. (2010). Los tesoros de los mallku de Pocona y Totora en el siglo XVI. Chungara $42(1): 199-220$. 
»Diccionario (1936). Diccionario de la Lengua Española. Real Academia Española. Madrid, Talleres Espasa Calpe.

» González, A. R. (1982). Las “provincias” incas del antiguo Tucumán. Revista del Museo Nacional XLVI: 317-380, Lima.

» González, A. R. (1992). Las placas metálicas de los andes del sur. Berlín, Kommision für Allgemaine und Vergleinchende Archaologie-Zabern 46.

» González Holguin, D. (1608). Diccionario castellano-quechua/ quechua /castellano [online]. Disponible en Internet: www.lenguandina.org. Consultado el 15 de octubre de 2011.

» González, L. y G. Gluzman (2007). Innovación y continuidad en la metalurgia del Noroeste Argentino. El caso del Bronce. Mundo de Antes 5: 187-210.

» González, A. R. y V. Núñez Regueiro (1968-1969). Ensayo sobre los tensores y manoplas del N. O. argentino. Boletín del Museo Nacional de Historia Natural XXX: 237-290. Santiago de Chile.

» Guaman Poma de Ayala ([1615] 1980). El primer nueva corónica y buen gobierno. Edición crítica de Murra J. y R. Adorno. México, Siglo XXI.

» Hogg, A. G.; Hua, Q.; Blackwell, P.; Niu, M.; Buck, C. E.; Guilderson, T. P.; Heaton, T. J.; Palmer, J. G.; Reimer, P. J.; Reimer, R. W.; Turney, C. S. y S. R. Zimmerman (2013). Radiocarbon 55 (4) 1889-1903 by the Arizona Board of Regents on behalf of the University of Arizona. DOI: 10.2458/azu_js_rc. 55_16783. Disponible en Internet: https://journals. uair.arizona.edu .Consultado el 28 de octubre 2018.

» Horta Tricallotis, H. (2008). “Insignias para la frente de los nobles Inca: Una aproximación Etnohistórica-Arqueológica al principio de dualidad” en González Carvajal, P. y T. Bray (eds.), Lenguajes visuales de los Incas. BAR International Series 1848: 71-89.

» lácona, A. y R. Raffino (1993). De Titicaca a Omahuaca durante el siglo XVI en Raffino, R. (ed.), Inka. Arqueología, historia y urbanismo del altiplano andino: 235-283. Buenos Aires, Editorial Corregidor.

»Lechtman, H. y A. Macfarlane (2006). “Bronce y redes de intercambio andino durante el Horizonte Medio: Tiwanaku y San Pedro de Atacama” en Lechtman, H. (ed.), Esferas de interacción prehistórica y fronteras nacionales modernas: los Andes sur centrales: 503-539. Lima, IEP-IAR.

» Lorandi, A. M. (1984). Pleito de Juan de Ochoa de Zárate por la posesión de los indios ocloyas. ¿Un caso de verticalidad étnica o un relicto de archipiélago estatal? Runa XIV: 123-142.

»Lozano, P. ([1733] 1941). Descripción corográfica del Gran Chaco Gualamba. Tucumán, Instituto de Antropología. Universidad Nacional de Tucumán. (Publicación 288).

» Martínez, J. L. (1994). Rituales fallidos, gestos vacíos: un desencuentro entre españoles y andinos en 1532. Mundo Precolombino. Revista del Museo Chileno de Arte Precolombino 1: 28-41.

» Martínez Garnica, A. (1989). Un caso de alteración aurífera en el bajo Magdalena. Boletín del Museo del Oro 23: 47-6o. Bogotá.

» Márquez Miranda, F. (1939). Cuatro viajes de estudio al más remoto Noroeste argentino. Revista del Museo de La Plata, Nueva Serie 1 (6): 93-243.

» Mayer, E. F. (1986). Armas y herramientas de metal prehispánicas en Argentina y Chile. Munich, Beck C. H.

» Meyers, A. e I. Combés (comps.) (2015). El fuerte de Samaipata. Estudios arqueológicos. 
Santa Cruz de la Sierra, Biblioteca del Museo de Historia, Universidad Autónoma Gabriel René Moreno.

» Morris, G. y A. von Hagen (2011). The Incas. Lords of the four Quarters. London, Thames and Hudson.

» Oliveto, L. G. (2011). “Ocupación territorial y relaciones interétnicas en los Andes meridionales. Tarija, entre los desafíos prehispánicos y temprano-coloniales”. Tesis Doctoral. Facultad de Filosofía y Letras, Universidad de Buenos Aires.

» Oliveto, L. G. y B. N. Ventura (2009). Dinámicas poblacionales de los valles orientales del sur de Bolivia y norte de Argentina, siglos XV-XVII. Aportes etnohistóricos y arqueológicos. Población y Sociedad 16: 119-150.

» Oliveto, L. G. y B. N. Ventura (2017). Final de la Jornada al Chaco de Ledesma Valderrama en 1631. Análisis y nuevas perspectivas a partir de documentación inédita. Relaciones de la Sociedad Argentina de Antropología XLII (2): 257-280.

"Owen, B. (2001). "The economy of metal and shell wealth goods” en D'Altroy, T. y C. Hastorf (eds.), Empire and Domestic Economy. Nueva York, Kluwer Academic Plenum Publishers.

"Owen, B. (2012). "The meaning of metals: The Inca and regional contexts of quotidian metal from Machu Picchu” en Burger R. y L. Salazar (eds.), The 1912 Peruvian Scientific Expedition Collections from Machu Picchu. Metal Artifacts: 91: 73-189. New Haven Ct, Yale University Publications in Anthropology.

»Palacios, T. y J. H. Rodríguez (1985). “Estudio metalúrgico de una pieza arqueológica” en Ventura, B., Metalurgia: Un aspecto poco conocido en la arqueología de las selvas occidentales. Informes de Investigación 2: 84-97. Buenos Aires, Programa de Estudios Prehistóricos, CONICET-UBA.

»Pärssinen, M. (1992). Tawantinsuyu: The Inca State and its Political Organization. Studia Historica 43. Helsinki, Societas Historica Finlandiae.

»Pärssinen, M. (2003). Tawantinsuyu: El Estado Inca y su organización política. Lima, IFEA.

»Pärssinen, M. y J. Kiriharju (2004). Textos andinos. Corpus de textos khipu incaicos y coloniales. Editores Acta Ibero-Americana 4. Fennica Series Hispano-americano 6, Instituto Iberoamericano de Finlandia y Departamento de Filología española y Facultad Complutense de Madrid.

» Pastells, P. (1915). Historia de la Compañía de Jesús en la Provincia del Paraguay (Argentina, Paraguay, Uruguay, Perú, Bolivia y Brasil). II. Madrid.

»Presta, A. M. (1997). “La población de los valles de Tarija, Siglo XVI. Aportes para la solución de un enigma etnohistórico en la frontera incaica” en Lorandi, A. M. (comp.), El Tucumán colonial y Charcas I: 163-175. Buenos Aires, Facultad de Filosofía y Letras, Universidad de Buenos Aires.

» Reinhard, J. y M. C. Ceruti (2010). Inca rituals and sacred Mountains. A study of the world's highest archeological sites. Los Angeles, Cotsen Institute of Archaeology, University of California.

» Rolandi de Perrot, D. (1974) Un hallazgo de objetos metálicos en el área del río DonceIlas (Provincia de Jujuy). Relaciones de la Sociedad Argentina de Antropología VIII: 153-16o.

» Rostworowski, M. (2001). Obras completas. I Pachacutec Inca Yupanqui. Lima, Instituto de Estudios Peruanos.

» Rubiolo, D. (2003). Hoja Geológica 2366-II/2166-IV. La Quiaca, Provincias de Jujuy y Salta (1:250.000). Boletín 246. Buenos Aires, Instituto de Geología y Recursos mineralesSEGEMAR. 
» Saignes, T. (1985). Potosi et le sud bolivien selon une ancienne carte C.M.H.L.B. Caravelle 44:123-128.

"Salas, A. (1945). El Antigal de Ciénega Grande (Quebrada de Pumamarca, Provincia de Jujuy). Publicación del Museo Etnográfico V: Serie A. Buenos Aires, Facultad de Filosofía y Letras, Universidad de Buenos Aires.

»Sánchez, S. y G. Sica (1990). La frontera oriental de Humahuaca y sus relaciones con el Chaco. Bulletín del'Institut Francais d'Etudes Andines 19 (2): 469-497.

»Sarmiento de Gamboa, P. ([1572] 2012). Historia de los Inkas. Transcripción, estudio crítico del manuscrito, estudio preliminar y notas de A. A. Manzo. La Plata, Yahuar.

»Santillán, H. ([ca.1563] 1968). Relación del origen, descendencia, política y gobierno de los incas. Madrid, Biblioteca de autores españoles. Crónicas Peruanas de Interés Indígena, CCTX.

"Someda, H. (2004). Aproximación a la imagen real de “los incas de privilegio". Boletín de Arqueología 8: 31-42. Lima, PUCP.

» Torres, D. ([1609] 1927). "Primera carta del Padre Diego de Torres" en Cartas Anuas 16091614, Documentos para la historia argentina XIX. Buenos Aires, Instituto de Investigaciones Históricas, Facultad de Filosofía Y Letras, Universidad de Buenos Aires.

»Ventura, B. N. (1985). Metalurgia: un aspecto poco conocido en la arqueología de las Selvas Occidentales. Informes de Investigación 2: 7-81. Buenos Aires, Programa de Estudios Prehistóricos, CONICET-UBA.

»Ventura, B. N. (1999). “Arqueología de los valles orientales a las Serranías de Zenta y Santa Victoria, Salta”. Tesis de doctorado. Facultad de Filosofía y Letras, Universidad de Buenos Aires.

»Ventura, B. N. (2001). “Los últimos mil años en la arqueología de las Yungas” en Berberián, E. y A. Nielsen (dirs.), Historia Argentina Prehispánica I: 447-492. Córdoba, Editorial Brujas.

»Ventura, B. N. (2016). “Esferas de interacción y circulación de bienes y poblaciones en un sector de la frontera oriental del Tawantinsuyu. Los valles del norte de Salta, Argentina" en Alconini, S. (ed.), Entre la vertiente tropical y los valles. Sociedades regionales e interacción prehispánicas en los Andes centro-sur: 301-318. La Paz, Editorial Plural.

»Ventura, B. N. y L. G. Oliveto (2014). Resabios de otros tiempos. Dominio incaico en los valles orientales del norte de Salta, Argentina. Bulletín de l'Institut Francais d' Etudes Andines 43 (2): 285-310.

»Ventura, B. N. y A. C. Scambato (2010). “Circulación de objetos de metal en las Yungas salteñas”. Ponencia presentada en el XVII Congreso Nacional de Arqueología Argentina. Instituto de Ciencias Humanas, Sociales y Ambientales (INCIHUSA-CONICET) y Facultad de Filosofía y Letras-Universidad Nacional de Cuyo. Mendoza, 11 al 15 de octubre.

»Ventura, B. N. y A. C. Scambato (2013). La metalurgia de los valles orientales del norte de Salta, Argentina. Boletín del Museo Chileno de Arte Precolombino 18 (1): 85-106.

»Ventura, B. N.; Becerra, M. F. y C. Angiorama (2016). “La producción minero-metalúrgica en la Puna de Jujuy y los valles orientales del norte de Salta (siglos XV-XVII). Un enfoque macro-regional”. Ponencia presentada en el XIX Congreso Nacional de Arqueología Argentina. Facultad de Ciencias Naturales e I. M. L., Universidad Nacional de Tucumán. San Miguel de Tucumán, 8 al 12 de Agosto.

»Vetter Parodi, L. (2013). “El Platero indio en los Andes: siglos XVI y XVII”. Tesis Doctoral. Escuela de Posgrado, Pontificia Universidad Católica del Perú.

»Vitry, C. (2014). Caminos y paisajes incas en los límites orientales del Tawantinsuyu. 
Ponencia presentada en las Primeras Jornadas de Etnohistoria, Arqueología y Antropología de la Macro región Sudeste de Bolivia (Tarija, Valles Interandinos, Chichas, Lipes), NO argentino y Norte de Chile. Museo de Paleontología y Arqueología de Tarija/ la Universidad de Bonn. Tarija, 10 al 13 de septiembre.

»Wachtel, N. (1982). "The mitimas of the Cochabamba valley. The colonization Policy of Huayna Capac” en Collier, G.; Rosaldo, R. yJ. Wirth (eds.), The Inca and Aztec States 14001800. Anthropology and History: 199-235. New York, Academic Press.

»Zanolli, C. E. (2008). Dos visitas coloniales a la jurisdicción de San Bernardo de la Frontera de Tarija. 1645-1659. Buenos Aires, Sección Etnohistoria. Facultad de Filosofía y Letras, Universidad de Buenos Aires. 